\title{
Ultra-late recurrence of non-small cell lung cancer over 10 years after curative resection
}

This article was published in the following Dove Press journal:

Cancer Management and Research

\author{
Dai Sonoda ${ }^{1,2}$ \\ Yosuke Matsuura' \\ Junji Ichinose' \\ Masayuki Nakao' \\ Hironori Ninomiya ${ }^{3}$ \\ Mingyon Mun' \\ Yuichi Ishikawa ${ }^{3}$ \\ Ken Nakagawa' \\ Yukitoshi Satoh ${ }^{2}$ \\ Sakae Okumura' \\ 'Department of Thoracic Surgical \\ Oncology, The Cancer Institute Hospital, \\ Japanese Foundation for Cancer Research, \\ Tokyo 135-8550, Japan; ${ }^{2}$ Department of \\ Thoracic Surgery, Kitasato University \\ School of Medicine, Sagamihara, Kanagawa \\ 252-0374, Japan; ${ }^{3}$ Department of \\ Pathology, The Cancer Institute Hospital, \\ Japanese Foundation for Cancer Research, \\ Tokyo I35-8550, Japan
}

Correspondence: Yosuke Matsuura Department of Thoracic Surgical Oncology, The Cancer Institute Hospital, Japanese Foundation for Cancer

Research, 3-8-3I, Ariake, Koto-ku, Tokyo I35-8550, Japan

Tel $+813352001 \mathrm{II}$

Fax +81335200141

Email yosuke.matsuura@jfcr.or.jp
Purpose: Most postoperative recurrences of non-small cell lung cancer (NSCLC) develop within 5 years after curative resection, with ultra-late recurrences developing over 10 years after the resection being rare. This study aimed to analyze the features of ultra-late recurrence in cases with NSCLC who had undergone curative resection.

Patients and methods: Among 1458 consecutive cases with NSCLC who underwent curative resection with systematic lymph node dissection during 1990-2006, 12 cases developed recurrence over 10 years after the resection. We defined the recurrence developing over 10 years after the resection as ultra-late recurrence and analyzed the factors related to it. Results: Among the 1458 cases, recurrence developed in 476 (32.6\%) cases. Of them, ultralate recurrence developed in 12 (2.5\%) cases. The ultra-late recurrence was histopathologically classified as adenocarcinoma in 11 cases and atypical carcinoid in 1 case. All cases were of invasive carcinoma. We compared ultra-late recurrence cases with non-recurrence cases and showed that none of the examined factors significantly influenced ultra-late recurrence; however, lymphatic invasion was close to significantly influencing it. There were two cases in which recurrence developed over 15 years after the resection; both cases were of adenocarcinoma with anaplastic lymphoma kinase (ALK) rearrangement.

Conclusion: There is a possibility of ultra-late recurrence developing over 10 years after the resection of any invasive NSCLC. Lymphatic invasion is close to significantly influencing ultra-late recurrence. Furthermore, a long follow-up period may be required in cases with adenocarcinoma with ALK rearrangement because it has the possibility of recurrence over 15 years after the resection.

Keywords: lung cancer, recurrence, resection, ALK rearrangement

\section{Introduction}

Complete surgical resection is the most effective treatment in patients with nonsmall cell lung cancer (NSCLC). However, recurrence remains a major obstacle in curing NSCLC. ${ }^{1-5}$

Most postoperative recurrences of NSCLC develop within 5 years after resection. Various studies have reported on the natural history of NSCLC within 5 years after resection; the prognostic factors after resection include pathologic stage, visceral pleural involvement, histology, vascular invasion, and need for pneumonectomy. $^{6-8}$

However, ultra-late recurrence developing over 10 years after resection is extremely rare. There are few case reports on ultra-late recurrence, and it is not well documented or well understood. ${ }^{9-15}$ In this study, we aimed to examine the factors related to ultra-late recurrence. 


\section{Materials and methods}

At the Cancer Institute Hospital, Japanese Foundation for Cancer Research, Tokyo, Japan, 1458 consecutive cases underwent lobectomy or more extensive resection complete with systematic lymph node dissection and achieved complete resection for primary NSCLC during January 1990December 2006. Complete resection was defined as cancernegative surgical margins both macroscopically and histologically. We investigated the postoperative recurrence of these cases as of January 2018.

After resection, patients were generally examined for 5 years at 6-month intervals; thereafter, they were followed up once a year until 10 years. The survival or death of patients who were lost to follow-up was confirmed via telephone or documentation with the patients or public office.

The follow-up evaluation included physical examination, chest radiography, and blood examination, including tumor marker evaluation. Moreover, chest computed tomography (CT) scans were performed yearly. Whenever any symptom or sign of recurrence was detected during these examinations, further evaluations were performed, including chest and abdomen $\mathrm{CT}$ scans, brain magnetic resonance imaging, bone scintigraphy, and positron emission tomography CT.

Recurrence was diagnosed on the basis of physical examination and diagnostic imaging findings, and was histologically and/or cytologically confirmed, when possible; the histological and/or cytological confirmation of recurrence was consistent with histopathological and immunohistochemical findings. Moreover, when possible, the mutational status of the epidermal growth factor receptor (EGFR) and anaplastic lymphoma kinase (ALK) rearrangement was confirmed in tumors (Figure 1).

The date of recurrence was defined as the date on which the recurrence was confirmed on the basis of imaging findings. The recurrence-free survival period was calculated from the day of resection to the date of the first recurrence or last living confirmation. The recurrence of NSCLC developing over 10 years after complete resection was defined as ultra-late recurrence.

Recurrence was distinguished from a second primary tumor following the criteria of Martini and Melamed. ${ }^{15}$ When the diagnosis was difficult, thoracic surgeons,

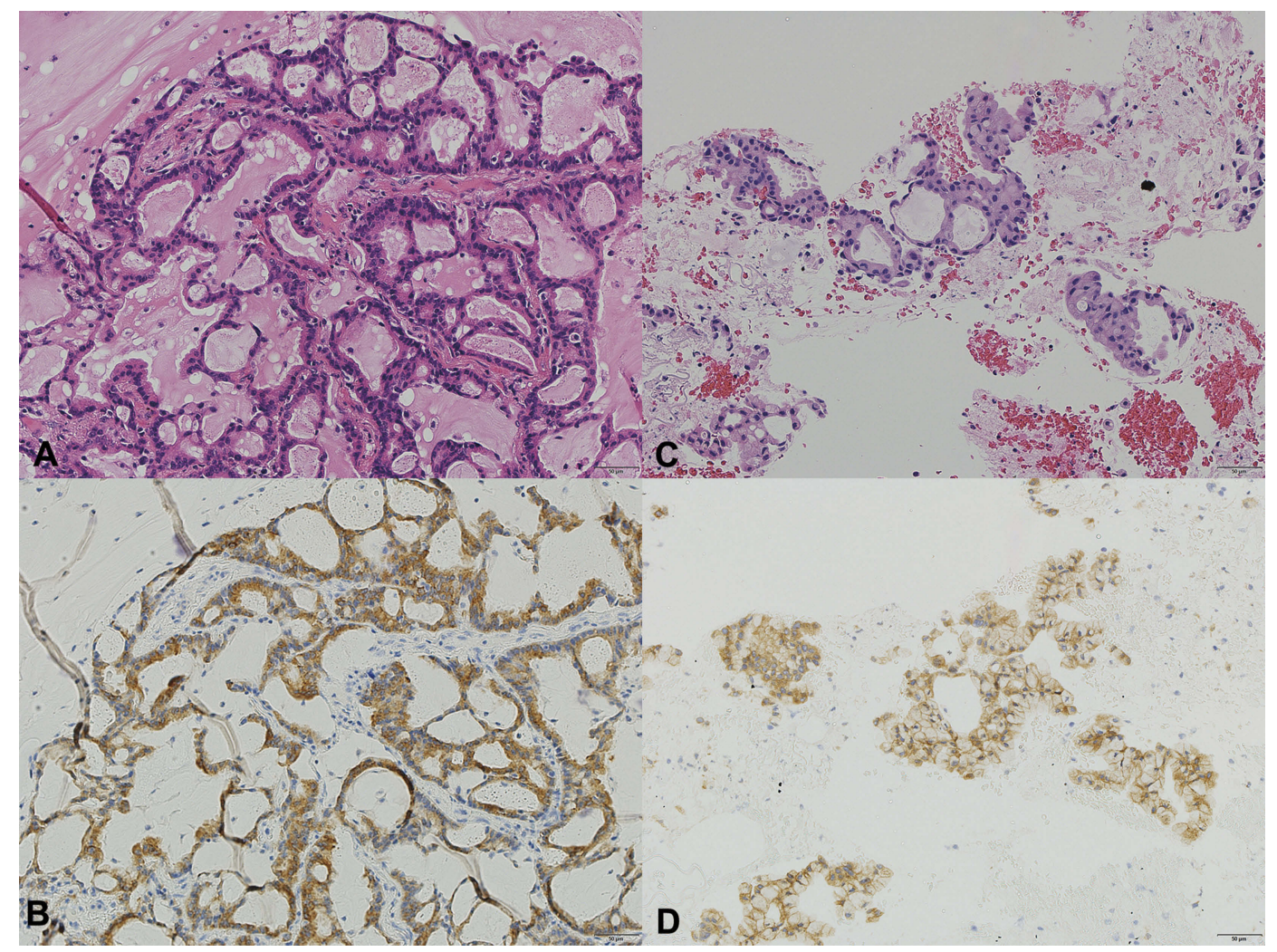

Figure I The pathological findings of the primary tumor and ultra-late recurrent tumor. In both the lesions, tumor cells showed glandular structures containing mucin (acinar adenocarcinoma). The histological findings of the primary and recurrent tumors are quite similar. (A) Hematoxylin and eosin staining of the primary tumor (20x). (B) Anti-anaplastic lymphoma kinase (ALK) antibody staining of the primary tumor (20x). (C) Hematoxylin and eosin staining of the recurrent tumor (20x). (D) Anti-anaplastic lymphoma kinase (ALK) antibody staining of the recurrent tumor (20x). 
respiratory physicians, radiologists, and pathologists conferred to decide whether the tumor was a recurrence or a second primary tumor.

Recurrence was classified into two categories: local recurrence and distant recurrence. Local recurrence was defined as the evidence of a tumor within the ipsilateral lung, ipsilateral pleural cavity, mediastinum, or bronchi. Distant recurrence was defined as the evidence of a tumor in the contralateral lung or outside the hemithorax.

The medical records of each patient were reviewed for clinicopathological information including age; sex; smoking habits and smoking index; pathological stage; lymph node metastasis; maximum tumor dimension in the resected specimen; histology; histological differentiation; and intratumoral lymphatic, vascular, or pleural invasion.

The tumor staging was determined according to the $7^{\text {th }}$ edition of the Union for International Cancer Control (UICC) for Cancer staging system; ${ }^{16}$ for cases of ultralate recurrence of NSCLC over 10 years after resection, the invasive size and staging was investigated according to the $8^{\text {th }}$ edition as well. ${ }^{17}$ Histological diagnoses were made on the basis of World Health Organization classification. ${ }^{18}$

Ultra-late recurrence cases were compared with nonrecurrence cases that could be followed up over 10 years after resection, and the factors related to ultra-late recurrence were analyzed.

Clinical characteristics and pathological factors were assessed using log-rank test. Multivariable analyses were performed using Cox's proportional hazards regression model. $P<0.05$ was considered as significant. Survival rate was calculated using Kaplan-Meier method. Analyses were performed using JMP pro version 14.0 (SAS Institute Inc., Cary, NC, USA) as windows statistical software.

This study was conducted in accordance with the amended Declaration of Helsinki. Written informed consent was obtained from each patient. The institutional review board of the Japanese Foundation for Cancer Research approved the study protocol (Approval No.2012-1042).

\section{Results}

Patients included 880 men and 578 women (median age, 64.0 years; age range: $16-86$ years). There were 489 nonsmokers and 969 smokers; the median smoking index was 570 . The extent of pulmonary resection comprised lobectomy, bilobectomy, and pneumonectomy in 1265, 111, and 82 cases, respectively.
The histological classification of the lung cancer was adenocarcinoma, squamous cell carcinoma, adenosquamous carcinoma, large cell carcinoma, carcinoid, and others in $70.4 \%$, $18.5 \%, 3.2 \%, 5.3 \%, 0.9 \%$, and $1.8 \%$ of the cases, respectively (Table 1).

The median follow-up period for all cases was 10.1 years from initial resection. The overall survival was $69.5 \%$ at 5 - years and $52.5 \%$ at 10 years.

Among 1458 cases, recurrence developed after complete curative resection in $476(32.6 \%)$ cases. Of them, recurrence developed within the first 5 years in 436 $(91.6 \%)$ cases, late recurrence developed at 5-10 years in $28(5.9 \%)$ cases, and ultra-late recurrence developed after 10 years in $12(2.5 \%)$ cases. The recurrence rate in cases with stage I, II, and III of the disease was $14.8 \%$, $39.6 \%$, and $64.7 \%$, respectively, indicating that the recurrence rate increased with the progression of the disease (Table 1).

A comparison of ultra-late recurrence cases with nonrecurrence cases showed that none of the examined factors significantly influenced ultra-late recurrence (Table 2); however, lymphatic invasion $(P=0.057)$ was close to significantly influencing it.

Table 3 presents the characteristics of the 12 cases $(5$ men, and 7 women; median age, 60 years; range, 49-70 years) in whom ultra-late recurrence developed. Most of these cases had a never to light smoking history (mean smoking index, 178). All cases had undergone lobectomy with systematic lymph node dissection.

Ultra-late recurrences developed 10.1-19.8 years (median, 12.1 years) after the resection. Of the 12 cases, 8 were judged to have pathological recurrences, and EGFR and ALK rearrangements in the tumor were confirmed in them. Molecular diagnosis could not be confirmed in one case because of the small biopsy specimen of the recurrent lesion; therefore, the case was judged to be recurrent on the basis of pathology and clinical course. Furthermore, of the 12 cases with ultra-late recurrence, 6 were of local recurrence and 6 of distant recurrence.

The histological classification was adenocarcinoma in 11 cases and atypical carcinoid in 1 case. All cases of adenocarcinoma were of invasive adenocarcinoma according to the $8^{\text {th }}$ edition of UICC for Cancer staging system. The pathological stage was described according to the $7^{\text {th }}$ and $8^{\text {th }}$ editions of UICC for cancer staging system. There were four cases of pathological $\mathrm{N}-0$, pl0, Ly0, and V0 and three cases of pathological N-1. 
Table I Clinicopathological characteristics of all I458 consecutive non-small cell lung cancer cases examined in this study

\begin{tabular}{|c|c|c|c|c|}
\hline Characteristics & $\begin{array}{l}\text { Non-recurrence, } \\
\text { n (\%) }\end{array}$ & $\begin{array}{l}\text { Early ( } \leq 5 \text { years) recur- } \\
\text { rence, } \mathrm{n}(\%)\end{array}$ & $\begin{array}{l}\text { Late }(5-10 \text { years) } \\
\text { recurrence, } n(\%)\end{array}$ & $\begin{array}{l}\text { Ultra-late ( } \geq 10 \text { years) } \\
\text { recurrence, } \mathbf{n}(\%)\end{array}$ \\
\hline Overall & 982 & 436 & 28 & 12 \\
\hline \multicolumn{5}{|l|}{ Age (years) } \\
\hline Median & 64 & 64 & 64 & 60 \\
\hline$\geq 65$ & $476(48.5)$ & $210(48.2)$ & $12(42.9)$ & $5(4 \mid .7)$ \\
\hline$<65$ & $506(5 I .5)$ & $226(51.8)$ & $16(57.1)$ & $7(58.3)$ \\
\hline \multicolumn{5}{|l|}{ Sex } \\
\hline Male & $582(59.3)$ & $275(63.1)$ & $18(64.3)$ & 5 (4I.7) \\
\hline Female & $400(40.7)$ & I6I (36.9) & $10(35.7)$ & $7(58.3)$ \\
\hline \multicolumn{5}{|l|}{ Smoking } \\
\hline Index median & 570 & 600 & 545 & 0 \\
\hline Non smoker & $340(34.6)$ & $130(29.8)$ & $12(42.9)$ & $7(58.3)$ \\
\hline Smoker & $642(65.4)$ & $306(70.2)$ & $16(57.1)$ & $5(4 \mid .7)$ \\
\hline \multicolumn{5}{|l|}{ Tumor size } \\
\hline$\leq 30$ & $570(58.0)$ & $|7|$ (39.2) & $10(35.7)$ & $5(4 I .7)$ \\
\hline$>30$ & $412(42.0)$ & $265(60.8)$ & $18(64.3)$ & $7(58.3)$ \\
\hline \multicolumn{5}{|l|}{$\mathrm{p}-\mathrm{N}$ status } \\
\hline No & 773 (78.7) & $159(36.5)$ & $18(64.3)$ & $8(66.7)$ \\
\hline $\mathrm{NI}$ & $123(12.5)$ & $99(22.7)$ & $6(21.4)$ & $4(33.3)$ \\
\hline N2 & $85(8.7)$ & $163(37.4)$ & $4(14.3)$ & 0 \\
\hline N3 & $I(0.1)$ & $15(3.4)$ & 0 & 0 \\
\hline \multicolumn{5}{|l|}{$\mathrm{p}$-stage } \\
\hline 1 & $654(66.6)$ & $93(21.3)$ & $15(53.6)$ & $6(50.0)$ \\
\hline II & $203(20.7)$ & $119(27.3)$ & $8(28.6)$ & $6(50.0)$ \\
\hline III & $125(12.7)$ & $224(5 I .4)$ & $5(17.9)$ & 0 \\
\hline \multicolumn{5}{|l|}{ Histologic type } \\
\hline Adenocarcinoma & $685(69.8)$ & $3 \mid 3(7 \mid .8)$ & $18(64.3)$ & II (9I.7) \\
\hline Squamous cell carcinoma & $202(20.6)$ & $62(14.2)$ & $5(17.9)$ & 0 \\
\hline Adenosquamous carcinoma & $20(2.0)$ & $26(6.0)$ & 0 & 0 \\
\hline Large cell carcinoma & $49(5.0)$ & $25(5.7)$ & $3(10.7)$ & 0 \\
\hline Carcinoid & II (I.I) & $\mathrm{I}(0.2)$ & 0 & I (8.3) \\
\hline Others & $15(1.5)$ & $9(2.1)$ & $2(7.1)$ & 0 \\
\hline \multicolumn{5}{|l|}{ Histologic differentiation } \\
\hline Well & $412(42.0)$ & $72(16.5)$ & $6(21.4)$ & $6(50.0)$ \\
\hline Moderate & $316(32.2)$ & $220(50.5)$ & $14(5.0)$ & $3(25.0)$ \\
\hline Poor & $149(15.2)$ & $78(17.9)$ & $4(14.3)$ & $2(16.7)$ \\
\hline Uncertain & $105(10.7)$ & $66(15.1)$ & $4(14.3)$ & I (8.3) \\
\hline \multicolumn{5}{|l|}{ Lymphatic invasion } \\
\hline 0 & $615(62.6)$ & $|5|(34.6)$ & $12(42.9)$ & $9(75.0)$ \\
\hline 1 & $95(9.7)$ & $|4|(32.3)$ & $10(35.7)$ & $3(25.0)$ \\
\hline Uncertain & $272(27.7)$ & $144(33.0)$ & $6(21.4)$ & 0 \\
\hline \multicolumn{5}{|l|}{ Vascular invasion } \\
\hline 0 & $417(42.5)$ & $65(14.9)$ & $7(25.0)$ & $6(50.0)$ \\
\hline 1 & $295(30.0)$ & $229(52.5)$ & $15(53.6)$ & $6(50.0)$ \\
\hline Uncertain & $270(27.5)$ & $142(32.6)$ & $6(2 \mid .4)$ & 0 \\
\hline
\end{tabular}

(Continued) 
Table I (Continued).

\begin{tabular}{|c|c|c|c|c|}
\hline Characteristics & $\begin{array}{l}\text { Non-recurrence, } \\
\text { n (\%) }\end{array}$ & $\begin{array}{l}\text { Early ( } \leq 5 \text { years) recur- } \\
\text { rence, } n(\%)\end{array}$ & $\begin{array}{l}\text { Late }(5-10 \text { years) } \\
\text { recurrence, } n(\%)\end{array}$ & $\begin{array}{l}\text { Ultra-late ( } \geq 10 \text { years) } \\
\text { recurrence, } \mathbf{n}(\%)\end{array}$ \\
\hline \multicolumn{5}{|l|}{ Pleural invasion } \\
\hline Absent & $76 \mid(77.5)$ & $23 \mid(53.0)$ & $23(82.1)$ & II (9I.7) \\
\hline Present & $221(22.5)$ & $205(47.0)$ & $5(17.9)$ & I (8.3) \\
\hline Uncertain & 0 & 0 & 0 & 0 \\
\hline \multicolumn{5}{|l|}{ Recurrence categories } \\
\hline Local & 0 & $105(24.1)$ & $6(2 \mid .4)$ & $6(50.0)$ \\
\hline Distant & 0 & 33। (75.9) & $22(78.6)$ & $6(50.0)$ \\
\hline
\end{tabular}

Furthermore, recurrence developed over 15 years after complete resection in two cases; both cases were of adenocarcinoma with ALK rearrangement.

\section{Discussion}

Postoperative recurrence of NSCLC commonly occurs within 5 years after resection; thus, NSCLC is often declared to be cured if no recurrence appears within 5 years after resection. However, postoperative recurrence of NSCLC over 5 years after resection has also been recognized. Several studies have reported recurrence of NSCLC over 5-10 years after resection.

About $4 \%-10 \%$ of the cases who underwent complete resection for stage I-III NSCLC had recurrence 5-10 years after resection. Some studies have shown nonclinicopathological factors to be the prognostic factors for the development of postoperative recurrence after 5-10 years, whereas others have shown intratumoral vascular invasion and lymph node metastasis to be the prognostic factors for the development of postoperative recurrence after $5-10$ years. ${ }^{1-3}$

Ultra-late recurrence of NSCLC over 10 years after curative resection is rare, and we could find only a few case reports of ultra-late recurrence ${ }^{9-15}$ (Table 4). However, there was no study that analyzed the features of ultra-late recurrence. To the best of our knowledge, this is the first study analyzing the features of ultra-late recurrence in patients who underwent complete resection for NSCLC.

Differential diagnosis between recurrent lung cancer and metachronous lung cancer remains controversial because there are no formally established differential criteria. ${ }^{1}$ Multiple intrapulmonary nodules are often diagnosed as intrapulmonary metastasis when they appear synchronously. However, in case of a single nodule, it is difficult to judge whether the nodule is intrapulmonary metastasis or a second primary tumor. In the present study, recurrence was distinguished from a second primary tumor following the criteria of Martini and Melamed. ${ }^{19}$

Ultra-late recurrence cases were compared with nonrecurrence cases, and it was observed that none of the examined factors significantly influenced ultra-late recurrence; however, lymphatic invasion was close to significantly influencing it. In addition, all cases that developed ultra-late recurrence had invasive carcinoma. Therefore, there is a possibility of ultra-late recurrence of any invasive NSCLC.

Furthermore, recurrence of NSCLC over 15 years after curative resection included adenocarcinoma with ALK rearrangement.

Adenocarcinoma with ALK rearrangement is usually found in younger patients and shows a favorable prognosis and clinical course after resection. ${ }^{20-22}$ However, there is a possibility of its recurrence even after a long interval. Studies have shown recurrence over 15 years after resection in cases with adenocarcinoma with ALK rearrangement. ${ }^{9,12-14}$ Therefore, a long follow-up period is recommended, particularly in patients with adenocarcinoma with ALK rearrangement.

This study has several limitations. First, we could not follow all the cases for 10 years or more; therefore, the cause of death is unclear in some cases. Of 835 deaths, the cause of death was not clear in 169 $(20.2 \%)$ cases. Therefore, there is a possibility that recurrence developed in these cases and was overlooked. Second, the follow-up period was not the same for all cases; therefore, patient selection bias and time trend bias may exist. Third, differential diagnosis between recurrent lung cancer and metachronous lung cancer has not been clearly established. Moreover, histopathological diagnosis could not be confirmed in all cases of recurrence. Therefore, there is a possibility that 
Table 2 Ultra-late recurrence compared with nonrecurrence that could be followed up over 10 years after resection

\begin{tabular}{|c|c|c|c|}
\hline Characteristic & Nonrecurrence, $\mathrm{n}(\%)$ & Ultra-late ( $\geq 10$ years) recurrence, $\mathbf{n}(\%)$ & Univariate $P$-Value \\
\hline Overall & 342 & 12 & \\
\hline \multicolumn{4}{|l|}{ Age (years) } \\
\hline$\geq 65$ & $103(30.1)$ & $5(4 I .7)$ & 0.162 \\
\hline$<65$ & $239(69.9)$ & $7(58.3)$ & \\
\hline \multicolumn{4}{|l|}{ Sex } \\
\hline Male & $184(53.8)$ & $5(4 I .7)$ & 0.241 \\
\hline Female & $158(46.2)$ & $7(58.3)$ & \\
\hline \multicolumn{4}{|l|}{ Smoking habits } \\
\hline Non smoker & $137(40.1)$ & $7(58.3)$ & 0.278 \\
\hline Smoker & $205(59.9)$ & $5(41.7)$ & \\
\hline \multicolumn{4}{|l|}{ Tumor size } \\
\hline$\leq 30$ & $220(64.3)$ & $5(4 I .7)$ & 0.966 \\
\hline$>30$ & $122(35.7)$ & $7(58.3)$ & \\
\hline \multicolumn{4}{|l|}{$\mathrm{p}-\mathrm{N}$ status } \\
\hline No & $279(81.6)$ & $8(66.7)$ & 0.431 \\
\hline $\mathrm{NI}-\mathrm{N} 3$ & $63(18.4)$ & $4(33.3)$ & \\
\hline \multicolumn{4}{|l|}{ p-stage } \\
\hline I & $244(71.3)$ & $6(50.0)$ & 0.430 \\
\hline II-III & 98 (28.7) & $6(50.0)$ & \\
\hline \multicolumn{4}{|l|}{ Histologic type } \\
\hline Adenocarcinoma & $267(78.1)$ & II (9I.7) & 0.595 \\
\hline Others & $75(21.9)$ & I (8.3) & \\
\hline \multicolumn{4}{|l|}{ Histologic } \\
\hline differentiation & $167(48.8)$ & $6(50.0)$ & $0.8 \mathrm{II}$ \\
\hline Well & $\mid 42(4 \mid .5)$ & $5(4 I .7)$ & \\
\hline Moderate-Poor & $33(9.6)$ & I (8.3) & \\
\hline \multicolumn{4}{|l|}{ Uncertain } \\
\hline \multicolumn{4}{|l|}{ Lymphatic invasion } \\
\hline 0 & $206(60.2)$ & $9(75.0)$ & 0.057 \\
\hline 1 & $27(7.9)$ & $3(25.0)$ & \\
\hline Uncertain & $109(31.9)$ & 0 & \\
\hline \multicolumn{4}{|l|}{ Vascular invasion } \\
\hline 0 & $149(43.6)$ & $6(50.0)$ & 0.875 \\
\hline I & $84(24.6)$ & $6(50.0)$ & \\
\hline Uncertain & $109(31.9)$ & 0 & \\
\hline \multicolumn{4}{|l|}{ Pleural invasion } \\
\hline Absent & $28 I(82.2)$ & II (9I.7) & 0.794 \\
\hline Present & $61(17.8)$ & I (8.3) & \\
\hline Uncertain & 0 & 0 & \\
\hline
\end{tabular}

the case we considered being recurrence was not completely excludable. Further large clinical prospective studies are needed to overcome these limitations.

\section{Conclusion}

There is a possibility of ultra-late recurrence developing over 10 years after the resection of any invasive NSCLC. 


\begin{tabular}{|c|c|c|c|c|c|c|c|c|c|c|c|c|}
\hline 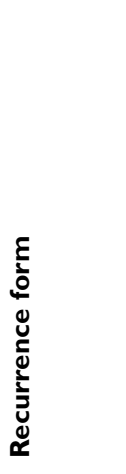 & 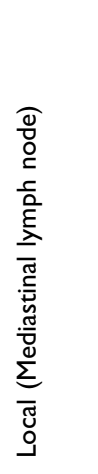 & 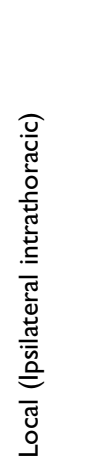 & 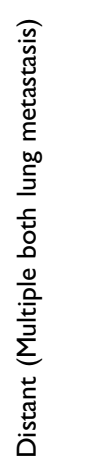 & 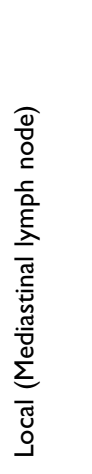 & 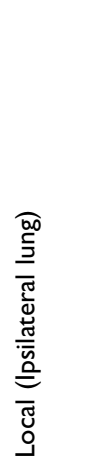 & 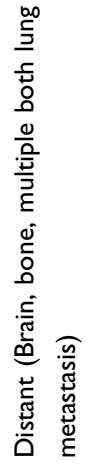 & 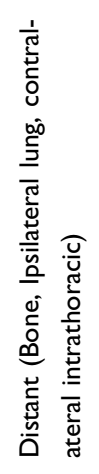 & 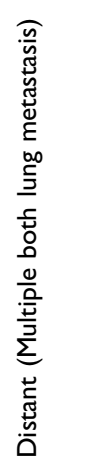 & 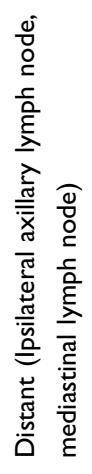 & 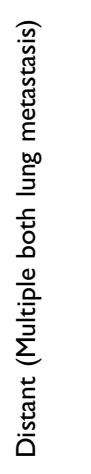 & 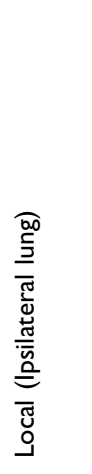 & 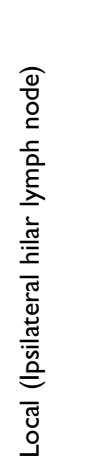 \\
\hline 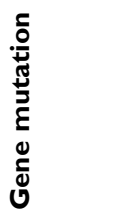 & $\begin{array}{l}\text { Ĩ } \\
\text { Zे }\end{array}$ & $\begin{array}{l}0 \\
\text { zo } \\
z\end{array}$ & $\begin{array}{l}0 \\
\text { zo } \\
z\end{array}$ & 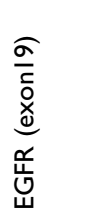 & $\frac{0}{\tilde{0}}$ & 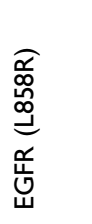 & 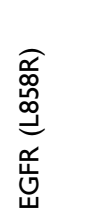 & 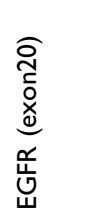 & $\begin{array}{l}0 \\
\stackrel{0}{0} \\
z\end{array}$ & $\begin{array}{l}\frac{5}{3} \\
0 \\
\frac{5}{5} \\
5\end{array}$ & 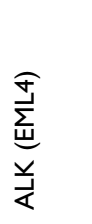 & 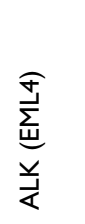 \\
\hline 窟 & 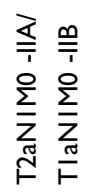 & 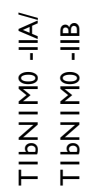 & 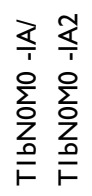 & 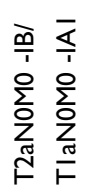 & 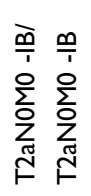 & 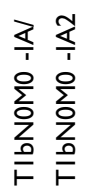 & 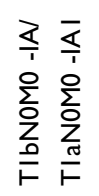 & 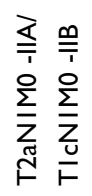 & 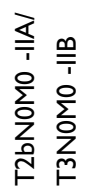 & 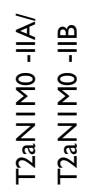 & 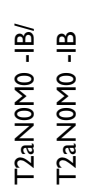 & 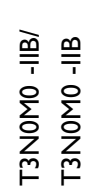 \\
\hline$\sum_{\lambda}^{\bar{a}}$ & $\stackrel{\varrho}{\frac{\partial}{o}}$ & $\stackrel{\varrho}{\frac{\partial}{\partial}}$ & 옹 & 옹 & $\frac{\varrho}{\partial}$ & 옹 & ঃ & ঃ & $\leqq$ & $\stackrel{\varrho}{\Xi}$ & $\frac{\varrho}{a}$ & $\stackrel{ }{\varrho}$ \\
\hline 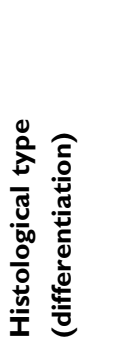 & 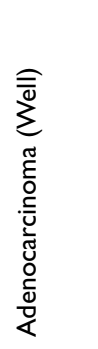 & 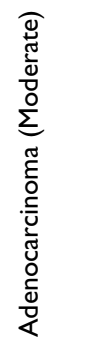 & 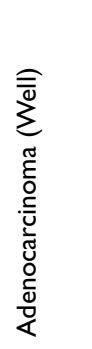 & 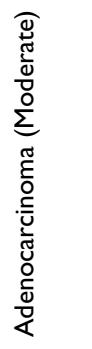 & 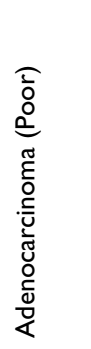 & 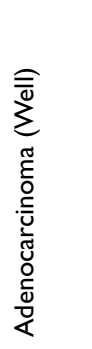 & 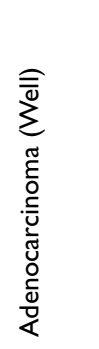 & 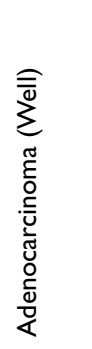 & 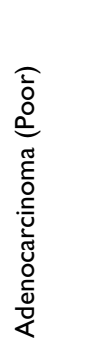 & 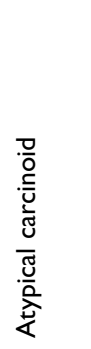 & 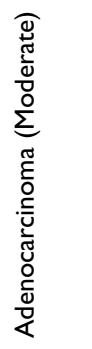 & 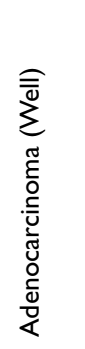 \\
\hline 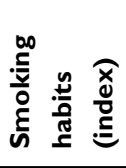 & 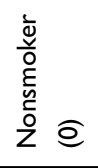 & 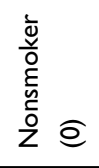 & 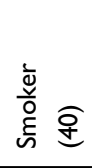 & 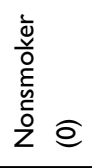 & 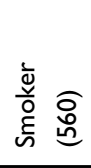 & 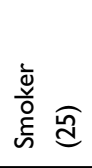 & 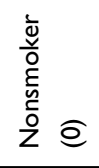 & 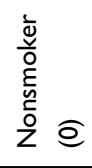 & 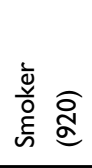 & 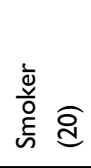 & 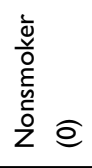 & 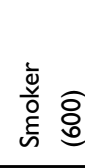 \\
\hline 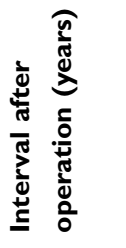 & $\overline{\underline{0}}$ & $\overline{\underline{O}}$ & $\stackrel{m}{0}$ & $\stackrel{\check{0}}{0}$ & $\stackrel{+}{=}$ & $\stackrel{\stackrel{\circ}{=}}{=}$ & $\stackrel{\stackrel{\varrho}{\underline{I}}}{ }$ & $\stackrel{\circ}{\underline{m}}$ & $\stackrel{\stackrel{\leftrightarrow}{m}}{\underline{m}}$ & 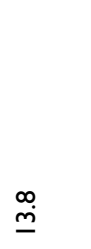 & $\stackrel{\infty}{\Perp}$ & $\stackrel{\infty}{\stackrel{\infty}{\varrho}}$ \\
\hline ڤ્ & 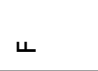 & ч & $\Sigma$ & ч & ч & $\Sigma$ & ч & ч & $\Sigma$ & $\Sigma$ & ч & $\Sigma$ \\
\hline 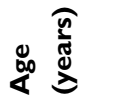 & $\overline{6}$ & R & P & P & q & $\stackrel{\infty}{n}$ & மீ & ô & นก & 요 & q & นn \\
\hline ฉ & - & $N$ & $m$ & $\sigma$ & in & o & $\wedge$ & $\infty$ & $\sigma$ & 으 & $=$ & $\simeq$ \\
\hline
\end{tabular}




\begin{tabular}{|c|c|c|c|c|c|c|}
\hline 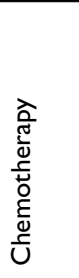 & 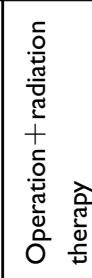 & 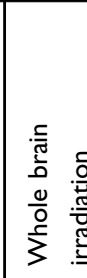 & 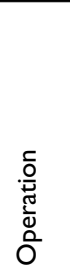 & 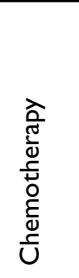 & 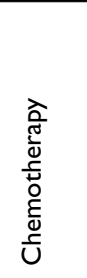 & 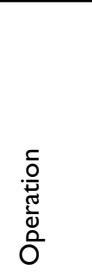 \\
\hline $\begin{array}{l}\text { 莒 } \\
\text { 畜 }\end{array}$ & 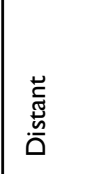 & 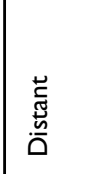 & ভ্ّ & 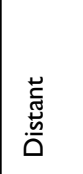 & ভ & 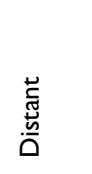 \\
\hline 并㞼 & 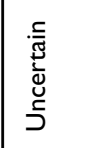 & 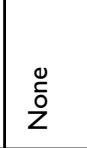 & 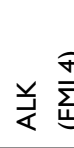 & 美籴 & 美㞼 & $\begin{array}{l}\stackrel{0}{0} \\
\stackrel{5}{z}\end{array}$ \\
\hline 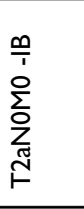 & 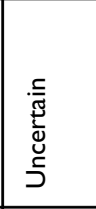 & 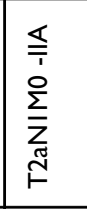 & 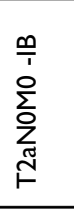 & 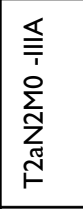 & 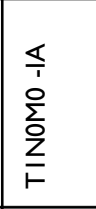 & 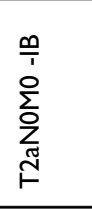 \\
\hline $\bar{\vdots}$ & 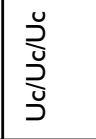 & 옹 & $\underset{\grave{S}}{\bar{S}}$ & ฏ্ড & 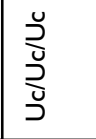 & $\overline{\grave{\partial}}$ \\
\hline 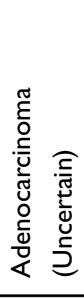 & 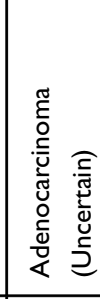 & 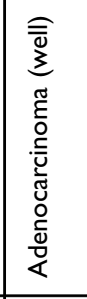 & 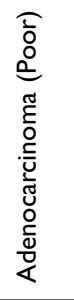 & 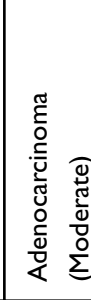 & 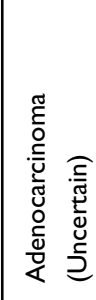 & 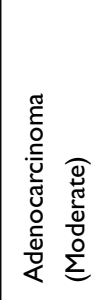 \\
\hline 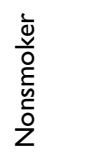 & $\breve{J}$ & \begin{tabular}{|l}
$\overline{\bar{\nu}}$ \\
$\overline{\mathrm{o}}$ \\
$\underline{\underline{E}}$
\end{tabular} & 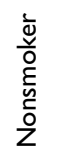 & 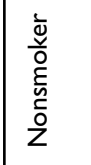 & 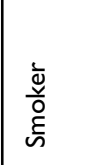 & 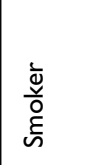 \\
\hline$\underline{\underline{a}}$ & 느 & $\subseteq$ & $\underline{\underline{n}}$ & $\underline{n}$ & $\simeq$ & $\underline{\sigma}$ \\
\hline${ }_{\Perp}$ & $\Sigma$ & $\Sigma$ & $\Sigma$ & $\Sigma$ & $\Sigma$ & $\Sigma$ \\
\hline o & in & 8 & q & q & 㐫 & $\overline{0}$ \\
\hline 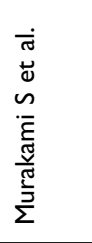 & 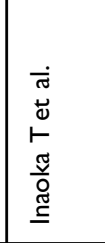 & 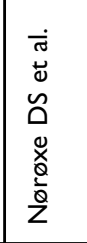 & 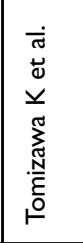 & 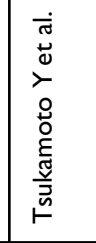 & 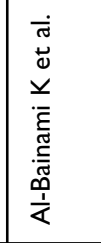 & 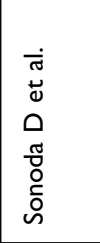 \\
\hline
\end{tabular}


Furthermore, lymphatic invasion is close to significantly influencing ultra-late recurrence.

A long follow-up period may be required in cases with adenocarcinoma with ALK-rearrangement because there is a possibility of its recurrence over 15 years after resection.

\section{Abbreviation list}

NSCLC, non-small cell lung cancer; ALK, anaplastic lymphoma kinase; CT, computed tomography; EGFR, epidermal growth factor receptor; UICC, Union for International Cancer Control.

\section{Acknowledgments}

This research did not receive any specific grant from funding agencies in the public, commercial, or not-for-profit sectors.

\section{Disclosure}

The authors report no conflicts of interest in this work.

\section{References}

1. Maeda R, Yoshida J, Hishida T, et al. Late recurrence of non-small cell lung cancer more than 5 years after complete resection. Chest. 2010;138:145-150. doi:10.1378/chest.09-2361

2. Okada M, Nishio W, Sakamoto T, Harada H, Uchino K, Tsubota N. Long-term survival and prognostic factors of five-year survivors with complete resection of non-small cell lung carcinoma. $J$ Thorac Cardiovasc Surg. 2003;126:558-562.

3. Martini N, Rusch VW, Bains MS, et al. Factors influencing ten-year survival in resected stages I to IIIA non-small cell lung cancer. J Thorac Cardiovasc Surg. 1999;117:32-38.

4. Taylor MD, Nagji AS, Bhamidipati CM, et al. Tumor recurrence after complete resection for non-small cell lung cancer. Ann Thorac Surg. 2012;93:1813-1821. doi:10.1016/j.athoracsur.2012.03.031

5. Martini N, Bains MS, Burt ME, et al. Incidence of local recurrence and second primary tumors in resected stage I lung cancer. $J$ Thorac Cardiovasc Surg. 1995;109:120-129. doi:10.1016/S0022-5223(95) 70427-2

6. Al-Kattan K, Sepsas E, Fountain SW, Townsend ER. Disease recurrence after resection for stage I lung cancer. Eur J Cardiothorac Surg. 1997;12:380-384. doi:10.1016/s1010-7940(97)00198-x

7. Martin J, Ginsberg RJ, Venkatraman ES, et al. Long-term results of combined-modality therapy in resectable non-small-cell lung cancer J Clin Oncol. 2002;20:1989-1995. doi:10.1200/JCO.2002.08.092

8. Khan OA, Fitzgerald JJ, Field ML, et al. Histological determinants of survival in completely resected T1-2N1M0 nonsmall cell cancer of the lung. Ann Thorac Surg. 2004;77:1173-1178. doi:10.1016/j. athoracsur.2003.08.080
9. Murakami S, Yokose T, Saito H, et al. Recurrent EML4-ALKassociated lung adenocarcinoma with a slow clinical course. Lung Cancer. 2010;69:361-364. doi:10.1016/j.lungcan.2010.05.019

10. Inaoka $\mathrm{T}$, Takahashi $\mathrm{K}$, Aburano $\mathrm{T}$, et al. Spinal metastasis from lung cancer fifteen years after surgery presenting a pseudohemangioma appearance of the vertebra: a case report. Spine. 2010;35:E86-E89. doi:10.1097/BRS.0b013e3181aa21d5

11. Nørøxe DS, Sørensen JB. Ultra-late relapse with a single cerebellar metastasis 10 years after complete surgery for stage IIA non-small cell lung cancer (bronchioalveolar carcinoma). J Thorac Oncol. 2012;7:764-765. doi:10.1097/JTO.0b013e3182460fc9

12. Tomizawa K, Ito S, Suda K, et al. Solitary pulmonary metastasis from lung cancer harboring EML4-ALK after a 15-year disease-free interval. Lung Cancer. 2013;80:99-101. doi:10.1016/j. lungcan.2012.12.011

13. Tsukamoto Y, Kanamori K, Watanabe T, et al. Recurrence of lung adenocarcinoma after an interval of 15 years revealed by demonstration of the same type of EML4-ALK fusion gene. Pathol Res Pract. 2014;210:1112-1116. doi:10.1016/j.prp.2014.08.002

14. Al-Baimani K, Sekhon HS, Wheatley-Price P. Recurrence of anaplastic lymphoma kinase (ALK) positive adenocarcinoma after 17 years: case report. Cancer Treat Сотти. 2015;4:6-9. doi:10.1016/j.ctrc.2015.03.001

15. Sonoda D, Mikubo M, Shiomi K, Satoh Y. Complete resection of oligo-recurrence of stage I lung adenocarcinoma 19 years after surgery: report of a rare case. Ann Thorac Surg. 2017;103:e119-e120. doi:10.1016/j.athoracsur.2016.07.023

16. Postmus PE, Brambilla E, Chansky K, et al. The IASLC lung cancer staging project: proposals for revision of the $\mathrm{M}$ descriptors in the forthcoming (seventh) edition of the TNM classification of lung cancer. $J$ Thorac Oncol. 2007;2:686-693. doi:10.1097/ JTO.0b013e31811f4703

17. Rami-Porta R, Bolejack V, Giroux DJ, et al. The IASLC lung cancer staging project: the new database to inform the eighth edition of the TNM classification of lung cancer. $J$ Thorac Oncol. 2014;9:1618-1624. doi:10.1097/JTO.0000000000000334

18. Travis WD, Brambilla E, Muller-Hermelink HK, Harris CC; World Health Organization. Classification of Tumours: Pathology and Genetics of Tumours of the Lung, Pleural, Thymus and Heart. Berlin: Springer; 2004.

19. Martini N, Melamed MR. Multiple primary lung cancers. J Thorac Cardiovasc Surg. 1975;70:606-612.

20. Inamura K, Takeuchi K, Togashi Y, et al. EML4-ALK lung cancers are characterized by rare other mutations, a TTF-1 cell lineage, an acinar histology, and young onset. Mod Pathol. 2009;22:508-515. doi:10.1038/modpathol.2009.2

21. Shaw AT, Yeap BY, Mino-Kenudson M, et al. Clinical features and outcome of patients with non-small-cell lung cancer who harbor EML4-ALK. $J$ Clin Oncol. 2009;27:4247-4253. doi:10.1200/ JCO.2009.22.6993

22. Blackhall FH, Peters S, Bubendorf L, et al. Prevalence and clinical outcomes for patients with ALK-positive resected stage I to III adenocarcinoma: results from the European thoracic oncology platform lungscape project. $J$ Clin Oncol. 2014;32:2780-2787. doi:10.1200/JCO.2013.54.5921 


\section{Publish your work in this journal}

Cancer Management and Research is an international, peer-reviewed open access journal focusing on cancer research and the optimal use of preventative and integrated treatment interventions to achieve improved outcomes, enhanced survival and quality of life for the cancer patient.
The manuscript management system is completely online and includes a very quick and fair peer-review system, which is all easy to use. Visit http://www.dovepress.com/testimonials.php to read real quotes from published authors.

Submit your manuscript here: https://www.dovepress.com/cancer-management-and-research-journal 